\title{
Fiber-Optic Surface Plasmon Resonance Sensor With Multi-Alternating Metal Layers for Biological Measurement
}

\author{
Yanjie WANG, Shengwei MENG, Yuzhang LIANG, Lixia LI, and Wei PENG* \\ College of Physics and Optoelectrical Engineering, Dalian University of Technology, Dalian, 116023, China \\ *Corresponding author: Wei PENGＥ-mail: wpeng@dlut.edu.cn
}

\begin{abstract}
Optical fiber based SPR sensors have attracted more and more attention due to their unique advantages over the prism-based SPR sensors. A novel fiber-optic SPR sensor with multi-alternating metal layers for biochemical analysis is presented in this paper. Based on the fundamental SPR theory of the fiber optic sensing technology, we theoretically investigated the effects of the existence of alternating layers deposited on sensing region SPR wavelength changes. The emphasis was placed on the numerical simulation of the fiber-optic SPR sensor's sensitivity which could be affected by its technical parameters such as the metal thickness, number of alternating layers. Results showed that, compared to the normal SPR sensor with the single metal layer, the proposed sensor had a wider detecting range of the refractive index and higher sensitivity, which can find applications in biological analysis.
\end{abstract}

Keywords: Surface plasmon resonance, fiber optics, biological detection, alternating metal layers

Citation: Yanjie WANG, Shengwei MENG, Yuzhang LIANG, Lixia LI, and Wei PENG, "Fiber-Optic Surface Plasmon Resonance Sensor With Multi-Alternating Metal Layers for Biological Measurement," Photonic Sensors, DOI: 10.1007/s13320-013-0119-2.

\section{Introduction}

Surface plasmon resonance (SPR) sensors have the wide application prospect in the field of chemical and biological analysis. Currently, most of the commercial SPR products are based on the optical prism structures like the Otto and Kretschmann configurations [1-3], Even, these SPR instruments have many advantages like the high sensitivity, multiple channel detection, still there are some limitations for applying these prism-based SPR sensors for in-situ industrial or environmental process monitoring. Optical fibers have inherent advantages, such as their fundamental compact structures, smaller sample volume requirement, and remote sensing capabilities. Compared to conventional prism-based SPR sensing devices, fiber-optic SPR sensors have recently drawn considerable attention because of the fundamentally simple structure, low cost, small sample volume, and amenability towards remote sensing applications [4-6]. Most fiber-optic SPR sensors are coated with the gold metal, which have been applied to the measurement depending on refractive index changes in most situations [5-8], even the SPR sensors with the silver film have the higher sensitivity, but the sensors that coated with the silver material are very easy to be oxidized during the application, which greatly restricts their application. In this paper, we present a novel multi-alternating metal layer film design for the fiber-optic SPR sensor. Compared to the traditional SPR sensor of the pure silver and gold

Received: 14 May 2013 / Revised version: 27 May 2013

(C) The Author(s) 2013. This article is published with open access at Springerlink.com 
film, respectively, it has the higher sensitivity and also can gradually avoid oxidized in the air, which can be used on fiber-optic sensor development for biological and biochemical analysis and monitoring.

\section{Theoretical model}

SPR is the collective oscillation of electrons in a solid or liquid stimulated by incident light. The resonance condition is established when the frequency of light photons matches the natural frequency of surface electrons oscillating against the restoring force of positive nuclei [6]. When a surface plasmon wave vector is equal to the evanescent wave vector, they will resonate, and the energy of incident light can be transferred to surface plasmons. There will be a resonance absorption in the reflectance spectrum, and the wavelength of the SPR dip increases with an increasing in the refractive index of the media attached to the film surface. The theoretical SPR wavelength can be predicted using a simulation that calculates the SPR reflection spectra for the three-layer or multi-layer structure sensor based on the Fresnel and Maxwell equations (9) and (10). The reflection ratio $R_{p}$ can be expressed as

$$
\begin{gathered}
R_{p}=\left|r_{p(0, N+1)}\right|^{2} \\
r_{p(k, N+1)}=\frac{r_{p(k, k+1)}+r_{p(k+1, N+1)} \exp \left(i 2 \varphi_{k+1}\right)}{1+r_{p(k, k+1)} r_{p(k+1, N+1)} \exp \left(i 2 \varphi_{k+1}\right)} \\
r_{p(k, k+1)}=\frac{n_{k+1} \cos \theta_{k}-n_{k} \cos \theta_{k+1}}{n_{k+1} \cos \theta_{k}+n_{k} \cos \theta_{k+1}}
\end{gathered}
$$

where $N$ is the number of media layers, $r_{p(0, n+1)}$ is the total reflection of P-polarized light, $r_{p(k, k+1)}$ is the reflection coefficient located on the surface between the two adjacent media, $\theta_{k}=\arcsin \left(n_{0} \sin \theta_{0} / n_{k}\right)$, and $\varphi_{k}=2 \pi d_{k} n_{k} \cos \theta_{k} / \lambda, k=1,2,3, \cdots, N$.

The above equations are suitable for the prism-based SPR sensors, while for the fiber-optic sensor, a theoretical model need to be modified. There are two transmission modes for light in the fiber, the meridional ray spreading along the meridional plane and the skew ray not passing through the meridional, which results in more sophisticated calculation. The effect of skew rays is weakened around the cylindrical surface of the optical fiber, and it can be negligible. After simplifying the model, we can only discuss the effects of meridional rays on the SPR phenomenon. Figure 1(a) illustrates a typical flat-tip fiber-optic SPR sensor with the gold film around the sensing area.

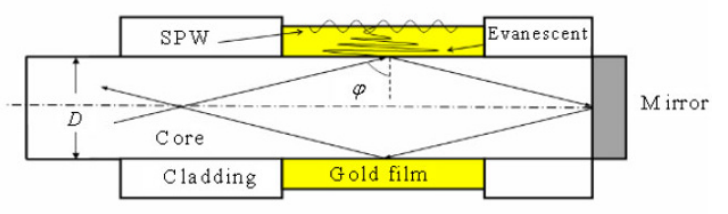

(a)

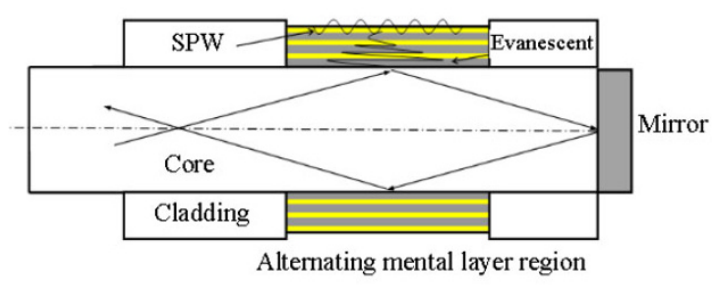

(b)

Fig. 1 Schematic of the typical fiber-optic SPR sensor (a) and fiber-optic SPR sensor with six alternating mental layers of silver and gold (b).

If the length of the sensing region is $L$, the number of reflection of lights within the sensing region is $N$ :

$$
N(L, D, \varphi)=\frac{L}{D \tan \varphi} .
$$

In the fiber sensors, angles of light cover from the critical angle $\varphi_{c}$ to $90^{\circ}$, which correspond to different transmission modes. The energy of every transmission mode is uneven, but all following the Gaussian distribution [5] as

$$
\begin{aligned}
& I(\alpha)=\left(108510+369.4 \alpha-1071 \alpha^{2}+\right. \\
& \left.133 \alpha^{3}-4.754 \alpha^{4}\right) / 103760 .
\end{aligned}
$$

Based on the above equations, we can get the total reflection of P-polarized light in the fiber sensor as

$$
\begin{gathered}
R_{p}\left(d, l, t, n_{2}(\lambda, t), n_{1}(\lambda)\right)=\frac{1}{M} \sum_{i=1}^{M} R^{N(L, D, \varphi)} I\left(\alpha_{i}\right) \\
\approx\left[\frac{1}{M} \sum_{i=1}^{M} I\left(\alpha_{i}\right)\right] R^{N(L, D, \varphi)} \propto R^{N(L, D, \varphi)}
\end{gathered}
$$


where $M$ is the number of the transmission mode, and the relation between $\alpha_{i}$ and $\varphi$ is $n_{0} \sin \alpha_{i}=n_{1} \sin \varphi, \varphi_{\mathrm{c}}<\varphi<90^{\circ} . R^{N(L, D, \varphi)}$, the total reflection of P-polarized light has the same change tendency, which allows making theoretical simulation by using lights of different incident angles to stimulate the SPR resonance phenomenon.

Equations (1) - (3) show that light reflectance of the SPR phenomenon relates to many factors including dielectric constants, the thickness of the metal, the numerical aperture of the optical fiber, and the refractive index of the fiber core. Silver and gold are the common metals used in SPR experiments. Silver could be gradually oxidized in the air and could not be kept for the long term or repeatable testing [11]. Therefore, even SPR sensors with the thin silver film possess the higher sensitivity, still due to the stable performance of the gold metal, and the gold coated fiber-optic SPR sensor has been widely applied in biochemistry, such as the self-assembly process of the thiol and gold substrate. To utilize both advantages of two metal materials and overcome their limitations in SPR testing, as shown in Fig. 1(b), we present a multi-alternating layers fiber-optic SPR sensor with a combined metal film of the silver gold metal theoretically in this paper.

\section{Simulation and results}

We made a series of simulation of the SPR spectrum to investigate the performance of the fiber-optic SPR sensor with multi-alternating metal layers. According to the SPR principle mentioned above, the characteristics of an SPR spectrum, such as the sensitivity and location of the SPR dip, are related to the length of the sensing region, the thickness of the material, the numerical aperture, and the diameter. For the typical terminated reflection-based fiber-optic SPR sensor, the large-core plastic optical fiber with the core diameter from $400 \mu \mathrm{m}$ to $600 \mu \mathrm{m}$, numerical aperture from 0.2 to 0.4 , is frequently used in experiments.
Using an incidence broadband light with the wavelength from $400 \mathrm{~nm}$ to $1200 \mathrm{~nm}$, the sensing region length of $5 \mathrm{~mm}$, and the core diameter of 400 $\mu \mathrm{m}$, the influence of the thickness of the metal and the number of alternating layers on the performance of the optical-fiber SPR sensor will be mainly discussed.

\subsection{Fiber-optic SPR sensor based on the three-layer structure}

In a typical terminated reflection-based fiber-optic SPR sensor, the surface plasmon wave is simultaneously excited at some angles. Contour plots of 3D simulations of the fiber-optic SPR sensor with the 50-nm-thickness silver or gold film for water between the SPR resonance wavelength and resonance angle in Fig. 2 present relations between them more clearly. Lights travel in the silica fiber with a numerical aperture of 0.37 adopted here from $75.6^{\circ}$ to $90^{\circ}$. It is clear that the SPR wavelength spreads from $531 \mathrm{~nm}$ to $578 \mathrm{~nm}$ and from $585 \mathrm{~nm}$ to $636 \mathrm{~nm}$, respectively. The detector gets the accumulation of all transmission modes corresponding to each angle. To simplify the process of the simulation calculation, the location of $\lambda_{\mathrm{SPR}}$ will be predicted for a given incident angle which is $85^{\circ}$ for theoretical analysis here to simplify the calculation procedure.

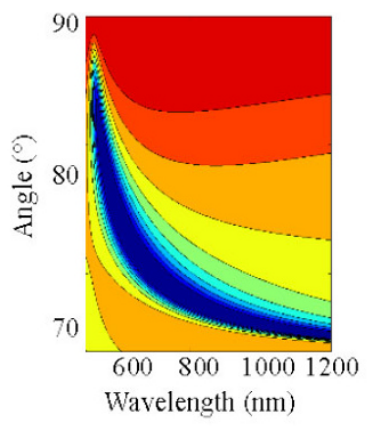

(a)

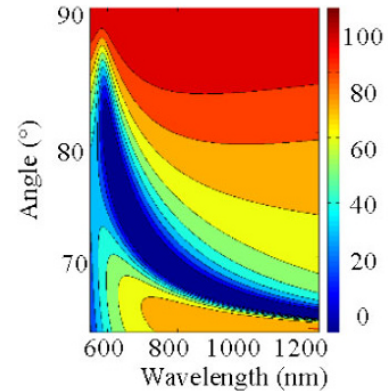

(b)
Fig. 2 Contour plots of the theoretical SPR spectra of the silver film (a) and gold film (b) for water (1.33) as the function of the incident angle and incident wavelength.

\subsection{Thickness of the metal layer}

Different thicknesses of the metal film are used 
to simulate the changes in SPR, as shown in Fig. 3. It can be concluded that the thickness of $35 \mathrm{~nm}-55$ $\mathrm{nm}$ of the sliver or gold film is suitable for the fiber-optic sensor because of the appropriate depth and width. Fiber-optic SPR sensors with the deeper resonance intensity have the enough anti-interference capability. Therefore, we will make the following simulations within this thickness range in this paper.

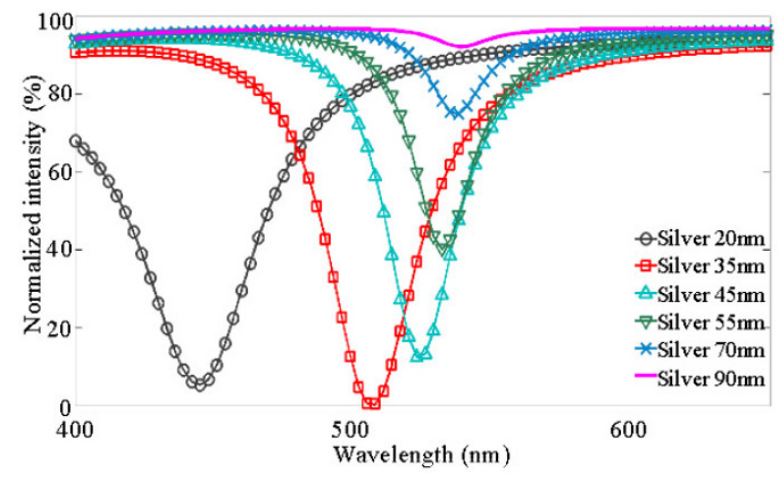

(a)

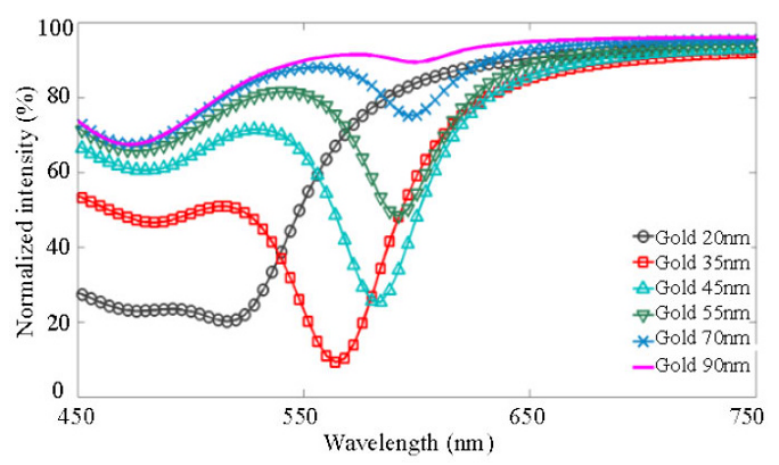

(b)

Fig. 3 SPR spectra with different thicknesses of the silver film (a) and gold film (b).

\subsection{Fiber-optic SPR sensor based on alternating mental layers}

To improve the performance of the SPR sensors for high sensitivity detection, a noble fiber-optic SPR sensor with multi-alternating metal layers is presented here. If the total thickness of the metal layers is same, the higher the purity of the silver film is, the higher the sensitivity of the SPR sensor is. Based on above discussions, we analyze a SPR sensor with the 45-nm-thickness metal film of 30-nm-thickness silver and 10-nm-thickness gold. If the number of the total metal layers is six, the thickness of the silver layer is $10 \mathrm{~nm}$, and the thickness of the gold layer is $5 \mathrm{~nm}$. The manufacture process of the typical fiber-optic SPR sensor based on three layers was previously described in [12]. And as to fabricate an alternating metal layer based SPR sensor, it is easier to develop the layers in a sputtering coater with two targets, such as the K575XD turbo sputter coater in our laboratory. The silver and gold materials can be sputtered on the fiber core turn to avoid the pollution and oxidation of the silver metal.

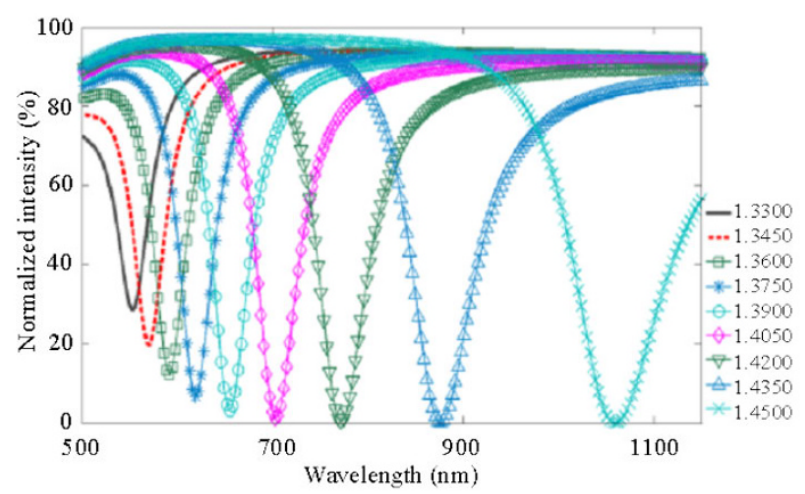

Fig. 4 SPR spectra for the fiber-optic sensor with ten alternating layers of silver and gold in relation to the refractive index.

We developed a Matlab program to calculate the SPR sensor with two, six, ten, and fourteen layers, respectively. The SPR spectra for the fiber-optic sensor with ten alternating layers of silver and gold in relation to the refractive index are shown in Fig. 4. Figure 5 describes the relations between SPR wavelengths and refractive index, which are fitted as follows:

$$
\begin{gathered}
\lambda_{\text {SPR }}=2892.9 \times n-3331.8 \\
\lambda_{\text {SPR }}=5.1486 \times 10^{6} n^{4}-2.8167 \times 10^{7} n^{3}+ \\
5.7794 \times 10^{7} n^{2}-5.2709 \times 10^{7} n+1.8028 \times 10^{7}
\end{gathered}
$$

where (7) is the linear function, and (8) is the 4th degree fitting function between the SPR wavelength and refractive index; $n$ is the refractive index, and the unit of the SPR wavelength is $\mathrm{nm}$.

Figure 6 illustrates the SPR spectra of the fiber-optic sensor with 45-nm-thickness gold and alternating layers of silver and gold for water (1.33). 
It shows that the SPR wavelength has a blue shift with an increasing in the number of layers, while $\lambda_{\text {SPR }}$ of fourteen layers moves towards the short wavelength about $20 \mathrm{~nm}$, compared to the two layers. However, the rate of wavelength movements increases slowly with an increase in the number of layers. Thus, the existence of alternating metal layers enlarges the detection range of media. More work about it will be done to realize a scheme for using this multilayer film on a dual or multiple channel fiber optic sensors.

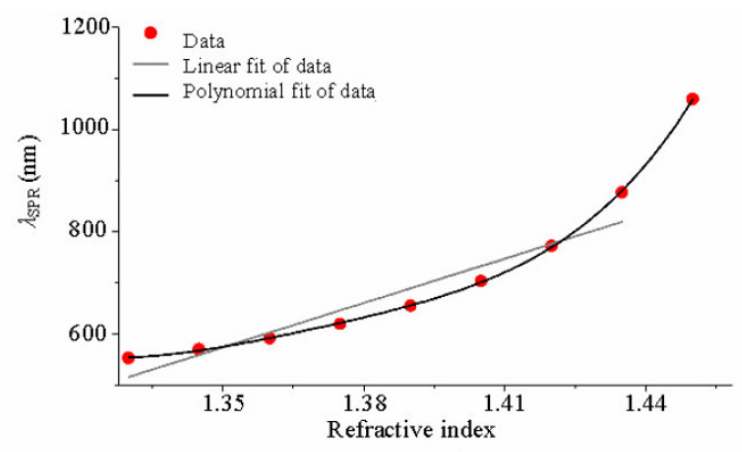

Fig. 5 Calibration curves of the refractive index and SPR wavelengths for the fiber-optic sensor with ten alternating layers.

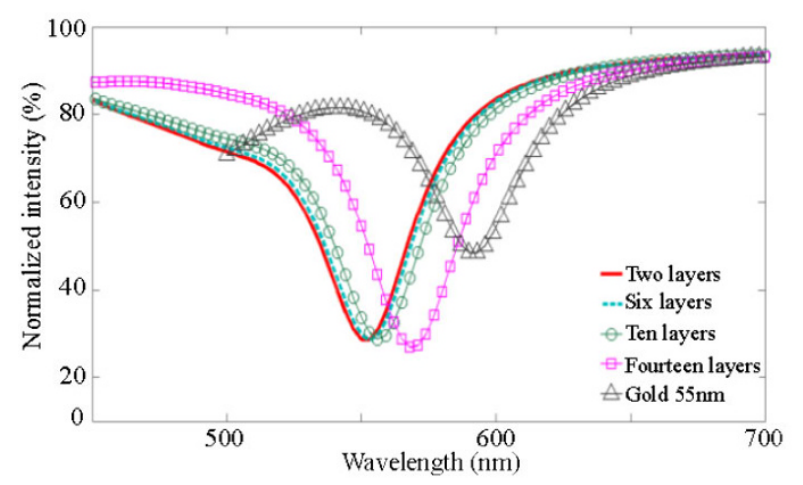

Fig. 6 SPR spectra for the fiber-optic sensor with different alternating layers.

\subsection{Sensitivity}

We also analyzed the sensitivity of the fiber-optic SPR sensor with alternating metal layers. Figure 7 shows that the sensitivity of the fiber-optic sensor with the 45-nm-thickness metal film of different numbers of layers increases from 2835 $\mathrm{nm} / \mathrm{RIU}$ to $2900 \mathrm{~nm} / \mathrm{RIU}$ with an increase in the number of alternating layers. More calculation work is done to study on the sensitivity of different thicknesses of alternating layers. With the same purity of silver and gold and the same alternating layer number, ten, the sensitivity is improved as the metal film thickness increases. As shown in Fig. 8, the sensitivity of alternating layers is lower than that of the pure silver film, but higher than that of the pure gold film. The alternating metal layers have the similar results with a gating based structure with the high sensitivity.

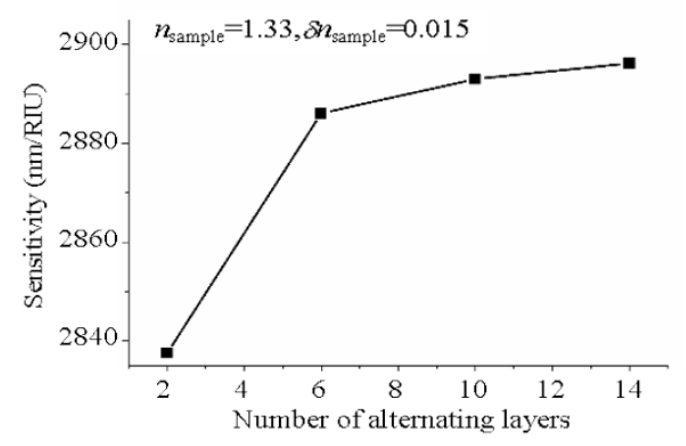

Fig. 7 Sensitivity distribution of the fiber-optic SPR sensor with the 45-nm-thickness metal film of different numbers of alternating layers.

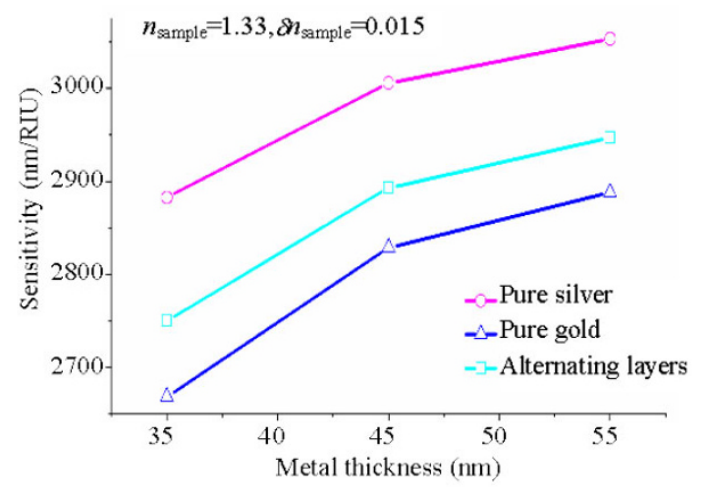

Fig. 8 Sensitivity distribution of the fiber-optic SPR sensor with different thicknesses of the sensing film based on the same refractive index.

In general, compared to the traditional SPR sensor of the pure gold film, the fiber-optic SPR sensor has the higher sensitivity which can deepen its application in biochemical analysis.

\section{Conclusions}

In summary, we made the theoretical 
investigation into the fiber-optic SPR sensor coated with alternating metal films by combining the traditional SPR theory with the fiber-optic sensing technology together. The simulation work demonstrated that compared to the gold or silver film coated single-layer based SPR sensor, the designed fiber-optic SPR sensor had advantages includes the wider detection range of refractive index detection and higher sensitivity, and it would be used in biochemistry wildly with the outer layer gold. The scheme that we constructed for the single-channel fiber-optic sensor could be adapted to the development of the dual-channel SPR sensor for the realization of the refractive index or temperature self-compensation. More researches and experiments are the under investigation to utilize the designed film on a high sensitivity dual-channel SPR sensor for the dual- or multiple-parameter measurement.

\section{Acknowledgment}

The authors would like to thank the financial supports from the National Nature Science Foundation of China (Grant Nos. 61137005 and 60977055) and the Ministry of Education of China (Grant Nos.NCET-09-0255 and SRFDP 20120041110040).

Open Access This article is distributed under the terms of the Creative Commons Attribution License which permits any use, distribution, and reproduction in any medium, provided the original author(s) and source are credited.

\section{References}

[1] R. J. Green, R. A. Frazier, K. M. Shakeshe, M. C. Davies, C. J. Roberts, and J. B. Tendler, "Surface plasmon resonance analysis of dynamic biological interactions with biomaterials," Biomaterials, vol. 21, no. 8, pp. 1823-1835, 2000.

[2] H. Lu, J. Homola, C. T. Campbell, G. G. Nenninger, S. S. Yee, and B. D. Ratner, "Protein contact printing for a surface plasmon resonance biosensor with on-chip referencing," Sensors and Actuators B: Chemical, vol. 74, no. 1-3, pp. 91-99, 2001.

[3] Y. Chena, B. Dong, and W. Zhou, "Surface plasmon resonance biosensor modified with multilayer silver nanoparticles films," Applied Surface Science, vol. 257, no. 3, pp. 1021-1026, 2010.

[4] J. Pollet, F. Delport, K. Janssen, K. Jans, G. Maes, H. Pfeiffer, et al.,"Fiber optic SPR biosensing of DNA hybridization and DNA-protein interactions," Biosensors and Bioelectronics, vol. 25, no. 4, pp. 864-869, 2009.

[5] K. S. Anuj and B. D. Gupta, "Absorption-based fiber optic surface plasmon resonance sensor: a theoretical evaluation," Sensors and Actuators B: Chemical, vol. 100, no. 3, pp. 423-431, 2004.

[6] W. Peng, S. Banerji, Y. Kim, and K. S. Booksh, "Investigation of dual-channel fiber-optic surface plasmon resonance sensing for biological applications," Optics Letters, vol. 30, no. 22, pp. 2988-2990, 2005.

[7] C. Lin, K. Chen, C. Hsiao, S. Lin, and C. Lee, "Design and fabrication of an alternating dielectric multi-layer device for surface plasmon resonance sensor," Sensors and Actuators B: Chemical, vol. 113, no. 1, pp. 169-176, 2006.

[8] A. K. Sharma and G. J. Moh, "Theoretical understanding of an alternating dielectric multilayer-based fiber optic SPR sensor and its application to gas sensing," New Journal of Physics, vol. 10, no. 2, pp. 023039, 2008.

[9] H. Li, Z. Jing, W. Peng, Q. Yu, W. Yao, L. Hu, et al., "Theoretical study of high-sensitivity surface plasmon resonance fiber optic sensing technology," in Proc. SPIE, vol. 8191, pp. 81911L, 2011.

[10] R. C. Jorgenson and S. S. Yee, "A fiber-optic chemical sensor based on surface plasmon resonance," Sensors and Actuators B: Chemical, vol. 12, no. 3, pp. 213-2220, 1993.

[11] M. Mitsushio, K. Miyashita, and M. Higo, "Sensor properties and surface characterization of the metal-deposited SPR optical fiber sensors with $\mathrm{Au}$, Ag, Cu, and Al," Sensors and Actuators A: Physical, vol. 125, no. 2, pp. 296-303, 2006.

[12] L. A. Obando, D. J. Gentleman, J. R. Holloway, and K S. Booksh, "Manufacture of robust surface plasmon resonance fiber optic based dip-probes," Sensors and Actuators B: Chemical, vol. 100, no. 3, pp. 439-449, 2004. 